\title{
Cortical Deafness Due to Ischaemic Strokes in Both Temporal Lobes
}

\author{
Magdalena Lachowska ${ }^{1}$, Agnieszka Pastuszka ${ }^{1}$, Jacek Sokołowski ${ }^{1}$, \\ Piotr Szczudlik², and Kazimierz Niemczyk ${ }^{1}$ \\ ${ }^{1}$ Department of Otorhinolaryngology Head and Neck Surgery, Medical University of Warsaw, Warsaw, Poland \\ ${ }^{2}$ Department of Neurology, Medical University of Warsaw, Warsaw, Poland
}

\section{Received June 2, 2020 \\ Revised July 18,2020 \\ Accepted September 15, 2020}

Address for correspondence

Magdalena Lachowska, MD, PhD

Department of Otorhinolaryngology

Head and Neck Surgery,

Medical University of Warsaw,

ul. Banacha 1a

02-097 Warszawa (Warsaw), Poland

Tel +48225992521

Fax +48225992523

E-mail mlachowska@wum.edu.pl
Cortical deafness is a clinical rarity whereby a patient is unresponsive to all types of sounds despite the preserved integrity of the peripheral hearing organs. In this study, we present a patient who suddenly lost his hearing following ischaemic infarcts in both temporal lobes with no other neurological deficits. The CT confirmed damage to the primary auditory cortex (Heschl's gyrus) of both hemispheres. Initially, the patient was unresponsive to all sounds, however, he regained some of the auditory abilities during 10 months follow up. Pure tone threshold improvement from complete deafness to the level of moderate hearing loss in the right ear and severe in the left was observed in pure tone audiometry. Otoacoustic emissions, auditory brainstem responses, and acoustic reflex findings showed normal results. The middle and late latency potential results confirmed objectively the improvement of the patient's hearing, however, after 10 months still, they were somewhat compromised on both sides. In speech audiometry, there was no comprehension of spoken words neither at 3 nor at 10 months. The absent mismatch negativity confirmed above mentioned comprehension deficit. The extensive auditory electrophysiological testing presented in this study contributes to the understanding of the neural and functional changes in cortical deafness. It presents the evolution of changes after ischaemic cerebrovascular event expressed as auditory evoked potentials starting from short through middle and long latency and ending with event-related potentials and supported by neuroimaging.

J Audiol Otol 2021;25(3):163-170

KEY WORDS: Evoked potentials · Hearing loss · Ischaemic stroke · Electrophysiology · Central nervous system.

\section{Introduction}

Cortical deafness is a clinical rarity whereby auditory signals cannot be perceived by the auditory cortex despite preserved integrity of the peripheral hearing organs, as mentioned by Silva, et al. [1]. In this condition, a patient is unresponsive to all types of sounds caused by lesions in the central auditory nervous system. This clinical entity is linked to four categories, as described by Musiek, et al. [2]. The first category involves a bilateral compromise of temporal lobes with extensive damage to the primary auditory cortex (Hes-

This is an Open Access article distributed under the terms of the Creative Commons Attribution Non-Commercial License (https://creativecommons.org/licenses/by-nc/4.0/) which permits unrestricted non-commercial use, distribution, and reproduction in any medium, provided the original work is properly cited. chl's gyrus). Other categories involve damage to subcortical structures associated with hearing that form connection between sensory and perceptual acoustic signal processing. The second and third categories involve unilateral temporal lobe damage or bihemispheric compromise of structures including the insula, inferior colliculus, cerebellum, posterior internal capsule, thalamic regions, and medial geniculate body, both combined with damaged Heschl's gyrus. Forth category involves damage to other areas associated with hearing such as inferior parietal lobe, supramarginal gyrus, anterior angular gyrus, inferior-frontal lobe, planum temporal also combined with damage to the primary auditory cortex.

Cortical deafness presents unique assessment challenges as well as treatment difficulties [3-7]. Even though pure tone audiometry shows complete deafness and there is no word 
reception in speech audiometry, the objective measures of peripheral auditory function are normal including acoustic reflexes and auditory brainstem responses (ABR). Patient recovery is unpredictable and usually the cortical deafness is transient and evolves with time into more selective hearing disorders [2,4,7-9].

In this study, we present a patient who suddenly and completely lost his hearing following ischaemic infarcts in both temporal lobes causing substantial damage to the primary auditory cortex with no other neurological deficits. The extensive auditory electrophysiological testing presented in this study and supported by neuroimaging contributes to the understanding of the evolution of the neural and functional changes in cortical deafness after the ischemic cerebrovascular event.

\section{Case Report}

\section{Ethical consideration}

The study was approved by the Local Institutional Ethics Committee where the study was conducted (AKBE/163/2018). The patient has given full written and informed consent, both for the participation and the publication of the report. The project conforms to The Code of Ethics of the World Medical Association (Declaration of Helsinki).

\section{Patient information}

A 42-year-old right-handed male with bilateral deafness referred to our department to diagnose his complete hearing loss. Three months earlier, he experienced complete hearing loss while watching TV. On the day of the incident, he was admitted to a hospital to neurology ward where left temporal lobe ischaemic stroke was diagnosed. CT revealed acute ischaemic stroke on the left side and former on the right. As it stays in the patient's medical documentation from that time, the physical examination did not reveal any neurological symptoms and signs except positive Babinski on the left side and bilateral deafness. The former stroke in the right temporal lobe was denied by the patient stating he did not experience any stroke before nor he underwent any neuro- or head imagining in the past. The patient did not have any symptoms at the time of the first ischemic event in the right temporal lobe, he did not notice any problems with hearing before. The old infarct in the right temporal lobe was an incidental finding. At the neurology ward where he was first admitted, due to the ischemic event and the serious cardiologic problems in the patient's medical history (described in detail in the later section named Medical history-comorbid diseases), the patient received the following medications: acenocoumarol (with prothrombin time derived measure International Normalized
Ratio control), digoxin, eplerenone, metoprolol, ramipril, torasemide. However, his hearing did not improve. He was discharged from the neurology ward and referred to an otolaryngology ward close to his hometown where he was diagnosed with bilateral deafness, suggested hearing aids, and referred to an audiologist in the outpatient department. The audiologist suggested cochlear implantation, however, the patient did not agree to proposed surgical treatment at that time. After three months, the patient referred to our department seeking help by himself due to his deafness.

\section{Testing protocol}

The medical history of the patient was taken along with otolaryngological and neurologic examination. The patient underwent extensive audiological testing including pure tone audiometry, speech audiometry, impedance audiometry, otoacoustic emissions, auditory brainstem evoked responses (ABR). The cortical deafness was suspected and auditory middle latency responses (MLR) and long latency responses (LLR), as well as mismatch negativity (MMN), were performed. Table 1 presents stimuli characteristics and signal acquisition parameters used for auditory electrophysiologic testing mentioned above. The patient was tested twice, first when he referred to our department the first time, which is three months after the stroke incident and second 10 months after the stroke as a follow-up.

The first and second CT scans were performed right after the stroke on the same day and three days later in the local neurology department close to where the patient was living and where he was admitted on the day of the cerebrovascular infarct incident. Ten months after the stroke again CT scans of the brain were performed in our department as the follow-up. MRI was not performed due to the implanted cardiac-pacemaker.

\section{Medical history-comorbid diseases}

Carefully taken medical history revealed surgery for transposition on the great arteries in early childhood, paroxysmal atrial fibrillation, cardiac-pacemaker implantation, thrombosis of the left subclavian vein, and veins of the lower limbs in the past, and Hepatitis $\mathrm{C}$ treated with interferon thirteen years ago.

\section{Clinical findings, diagnostic assessment, and therapeutic} intervention

At the time of three months after the stroke, when the patient referred to our department, the otorhinolaryngologic examination did not reveal anything specific other than bilateral deafness. The communicative contact with the patient at 
Table 1. Stimuli characteristics and signal acquisition parameters for the following auditory electrophysiological testing performed: auditory brainstem response (ABR), middle latency response (MLR), late latency response (LLR), and mismatch negativity (MMN)

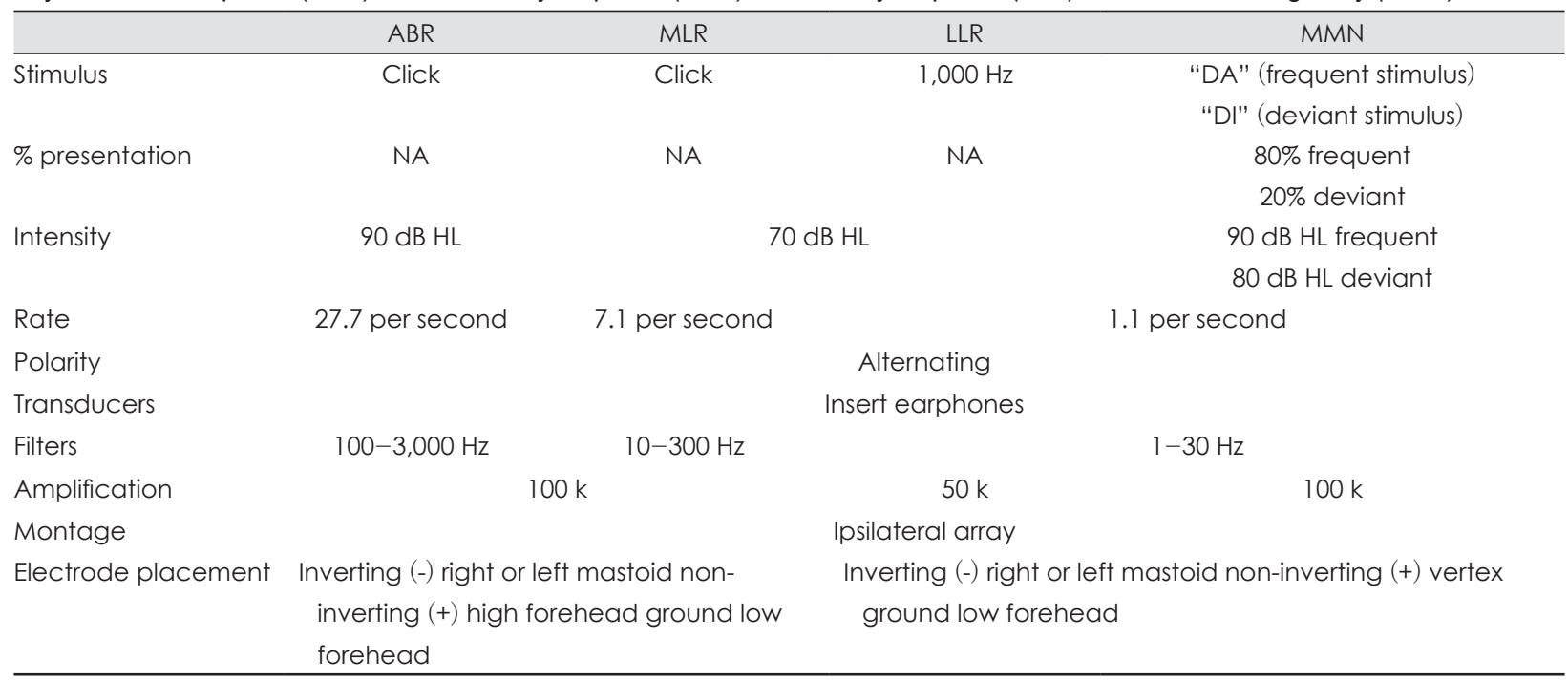

that time was difficult and presented a challenge. The patient claimed that he did not hear any sounds completely or understand spoken language. Although sometimes he seemed to understand what was being said to him because he answered questions adequately, however, a few seconds later he claimed he was unable to hear anything. Sometimes he tried to lip read, however, was not trained in it yet. The patient did not report any tinnitus at any time. The patient's speech was fluent and grammatically correct without any signs of aphasia. Orientation, calculations, and memory function were all intact. He followed written commands and answered written questions without any problems but presented big difficulties in maintaining his attention. The patient presented a changeable mood from cheerful to sad and aggressive sometimes. He was frustrated not to hear anything.

The pure tone audiometry was difficult to perform, the patient claimed he did not hear any sound presented to the ears and gave random answers. For speech audiometry, he did not repeat any monosyllabic word, again stating he was unable to hear anything (Fig. 1). Impedance audiometry revealed type A tympanograms on both sides with stapedial reflexes within 90-95 dB SPL. Distortion products otoacoustic emissions were registered at all tested frequencies from 1,000$6,000 \mathrm{~Hz}$ with sound-to-noise ratio (SNR) ranging from 6.5 to 30 . ABR presented regular morphologies with latencies and amplitudes within normal limits on both sides (Fig. 2).

MLR were inconclusive with Na-like waves on both sides with latencies around $20 \mathrm{~ms}$. The LLR showed a response on the left side with the latency of P1 around $75 \mathrm{~ms}$ and N1 around $110 \mathrm{~ms}$, and probable P1-N1 complex on the right side. The P1-N1 complex seemed to be compromised for both sides with wide $\mathrm{N} 1$ on the left and disturbed morphology of both P1 and N1 on the right. The MMN measured from the subtraction of two stimulus-evoked waveforms was absent on both sides (Fig. 2).

The extensive auditory electrophysiological testing supported by CT confirmed cortical deafness diagnosis. The patient was advised to attend psychological therapy and speech therapy to learn an alternative way of communicating with other people. He was also advised to use personal frequencymodulated (FM) systems [10]. His next follow-up visit was scheduled in the next seven months.

\section{Follow-up diagnostics and outcomes}

The patient followed the advised therapeutic intervention, however, he neglected the use of personal FM systems due to his economic problems. Ten months after the stroke the patient's hearing slightly improved. He was able to detect some sounds and claimed that sometimes he could understand some words, however, most of the time he just acknowledged hearing some sounds and experienced difficulties to identify the exact source or their exact nature. He was still unable to comprehend spoken words. He learned more to lip read, which was very helpful in his everyday life. The communicative contact with the patient was much better than 7 months earlier. The patient was much more peaceful and did not present such a changeable mood as before. He was more willing to cooperate during testing and maintaining attention was much better than the previous time.

The pure tone audiometry disclosed moderate hearing loss thresholds in the right ear and severe in the left (Fig. 1). For speech audiometry, the patient did not repeat any monosyl- 


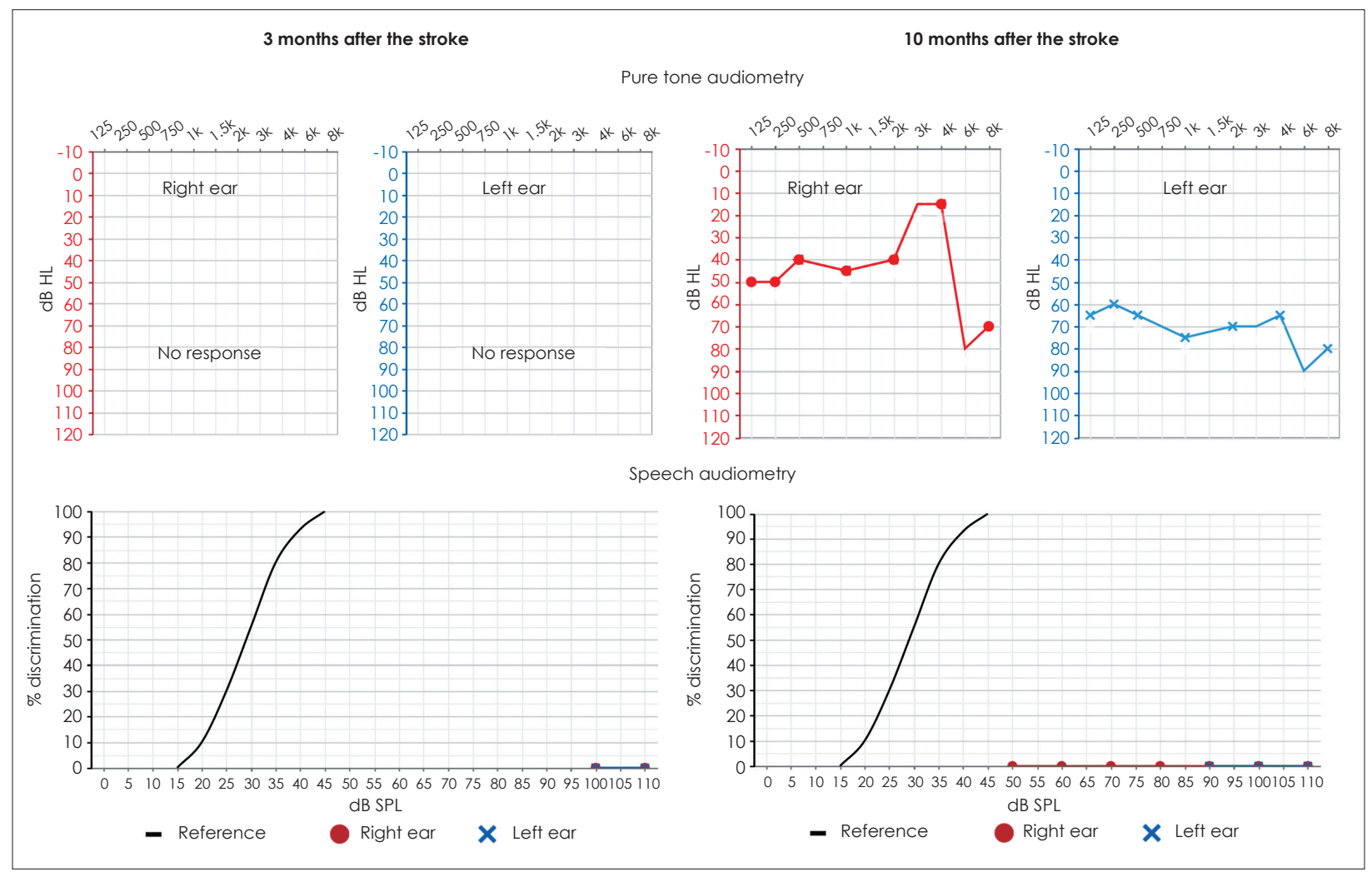

Fig. 1. Pure tone audiometry (first row) and monosyllabic speech audiometry (second row) results in a patient with cortical deafness 3 months (first column) and 10 months (second column) after a stroke of both temporal lobes. There are no thresholds detected at 3 months, and moderate hearing loss thresholds in the right ear and severe in the left at 10 months. In speech audiometry, the patient was not able to discriminate any presented monosyllabic word at any intensity neither 3 nor 10 months after the stroke.

labic words, claiming he just heard something unspecific, he was unable to understand the words. Impedance audiometry revealed type A tympanograms on both sides with stapedial reflexes within 85-95 dB SPL. Distortion products otoacoustic emissions were registered at all tested frequencies from 1,000-6,000 $\mathrm{Hz}$ with SNR ranging from 6.5 to 28 . ABR presented regular morphologies with latencies and amplitudes within normal limits on both sides (Fig. 2).

The MLRs were detected on both sides with robust $\mathrm{Na}$ and $\mathrm{Pa}$ waves and their latencies were about $25 \mathrm{~ms}$ and $45 \mathrm{~ms}$ on the left side, respectively, and $25 \mathrm{~ms}$ and $40 \mathrm{~ms}$ on the right, respectively (Fig. 2). The LLRs showed responses on both sides with P1 and N1 latencies around $75 \mathrm{~ms}$ and $125 \mathrm{~ms}$ on the left side, respectively, and $85 \mathrm{~ms}$ and $130 \mathrm{~ms}$ on the right, respectively. However, the LLRs seemed to be compromised with disturbed morphology of P1 and wide N1 on the left and wide N1 on the right. The MMN was absent on both sides in response to deviant stimuli (Fig. 2).

The patient was advised to continue attending psychological therapy and speech therapy to train more in the alternative way of communicating with other people and to train more his conscious awareness to sounds [11]. He was again advised to use personal FM systems [10], which he neglected the other time. The patient expressed his willingness and strong motivation to continue the proposed therapeutic intervention along with the use of FM systems because the outcomes so far have been encouraging for him.

\section{Radiological assessment}

First CT revealed (Fig. 3), in the right hemisphere, large hypodense lesion including transverse temporal gyrus of Heschl, auditory radiations, ranging up the superior parietal lobule-Brodmann area (BA) 41, 42, 40, 22, 5 and partial 3, 2, 1 , and in the left temporal region smaller hypodensity which included mostly transverse temporal gyrus of Heschl BA- 41. The second CT performed 3 days later showed the progression of hypodense changes in the left side with no contrast enhancement (Fig. 3), the lesion on the right side did not change. Control CT performed 10 months after the stroke revealed stable changes in the right hemisphere and clear hypodensity on the left temporal lobe (Fig. 3). 


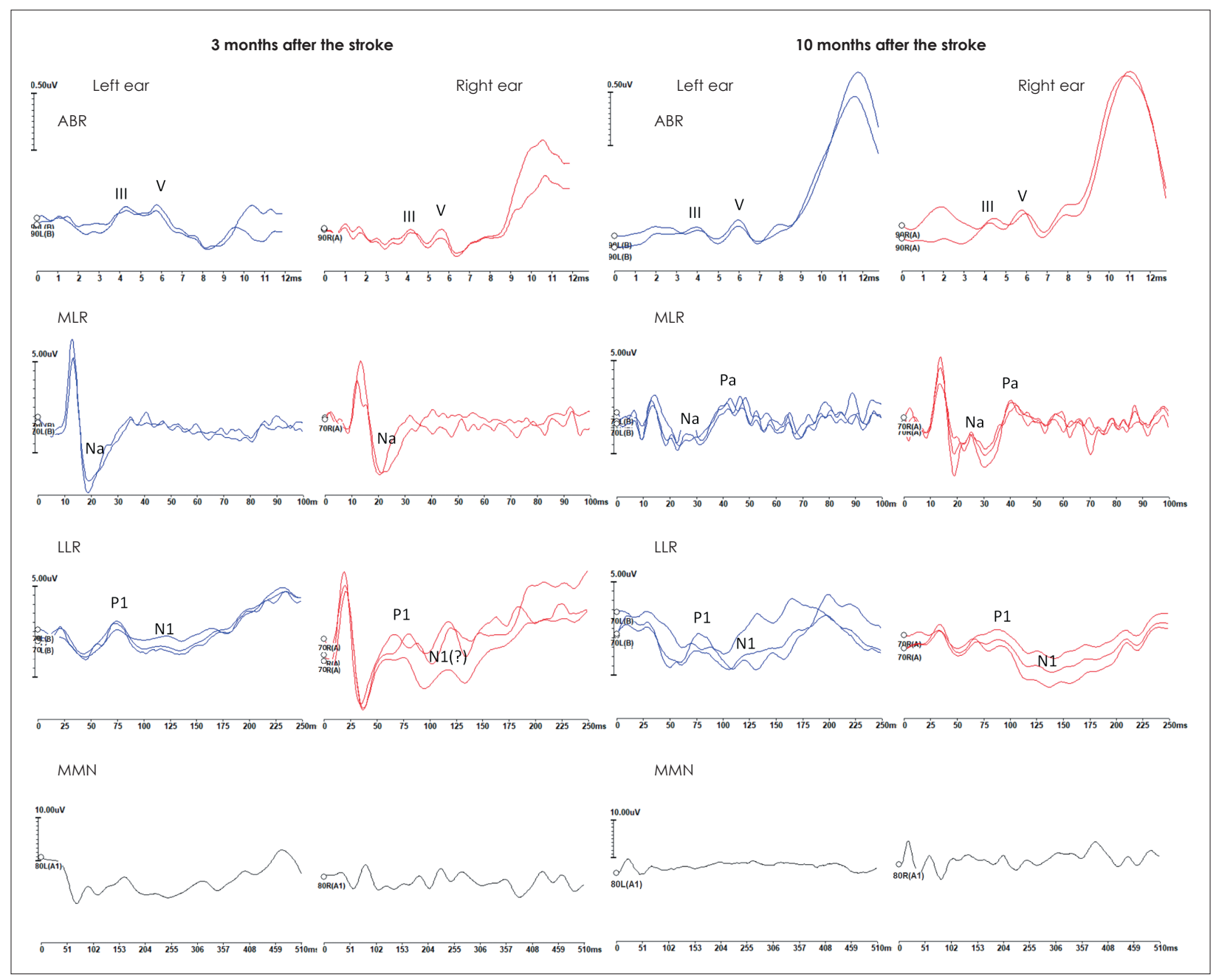

Fig. 2. ABR (first row), MLR (second row), LLR (third row), and MMN (fourth row) recorded in a patient with cortical deafness 3 months (first column) and 10 months (second column) after a stroke of both temporal lobes. The waves for each type of auditory evoked potential are marked where applicable. ABR: auditory brainstem responses, MLR: middle latency responses, LLR: long latency responses, MMN: mismatch negativity.

\section{Discussion}

Bilateral extensive damage of the primary auditory cortex and/or auditory radiations due to cerebrovascular ischemic events in both temporal lobes may result in a complete dramatic loss of hearing called cortical deafness [4-7,12], which was the case in the patient described in this study. Neuroanatomical changes found in our patient fall into the category of cortical deafness with the extensive primary auditory cortex (Heschl's gyrus) damage of both hemispheres as described by Musiek, et al. [2]. The patient denied any previous cerebrovascular event before the described acute one that caused complete deafness. The first ischaemic infarct in the right hemisphere was probably not clinically disclosed, even though it was quite massive, and the present neurologic examination revealed only positive Babinski on the left side. Neuroimag- ing performed on the day of the present stroke and after 10 months revealed previous and stable ischemic changes in the right hemisphere extending from transverse temporal gyrus to the parietal lobe but with partial sparing of primary sensory cortex. This could be clinically undetectable by the patient even though included the whole auditory cortex on the right side. Due to bilateral hearing pathways, the patient could not experience any noticeable hearing problems at that time.

The hypodense lesion in the left hemisphere observed in the first performed CT consisted of acute ischemic changes that looked more apparent on the second CT three days later. The lesion mostly affected the auditory cortex on the left. Ten months later the changes in the left temporal lobe became a little smaller probably because at the beginning the ischaemic core was surrounded by edema that looked alike in CT scans. 
CT scans on the day of the stroke
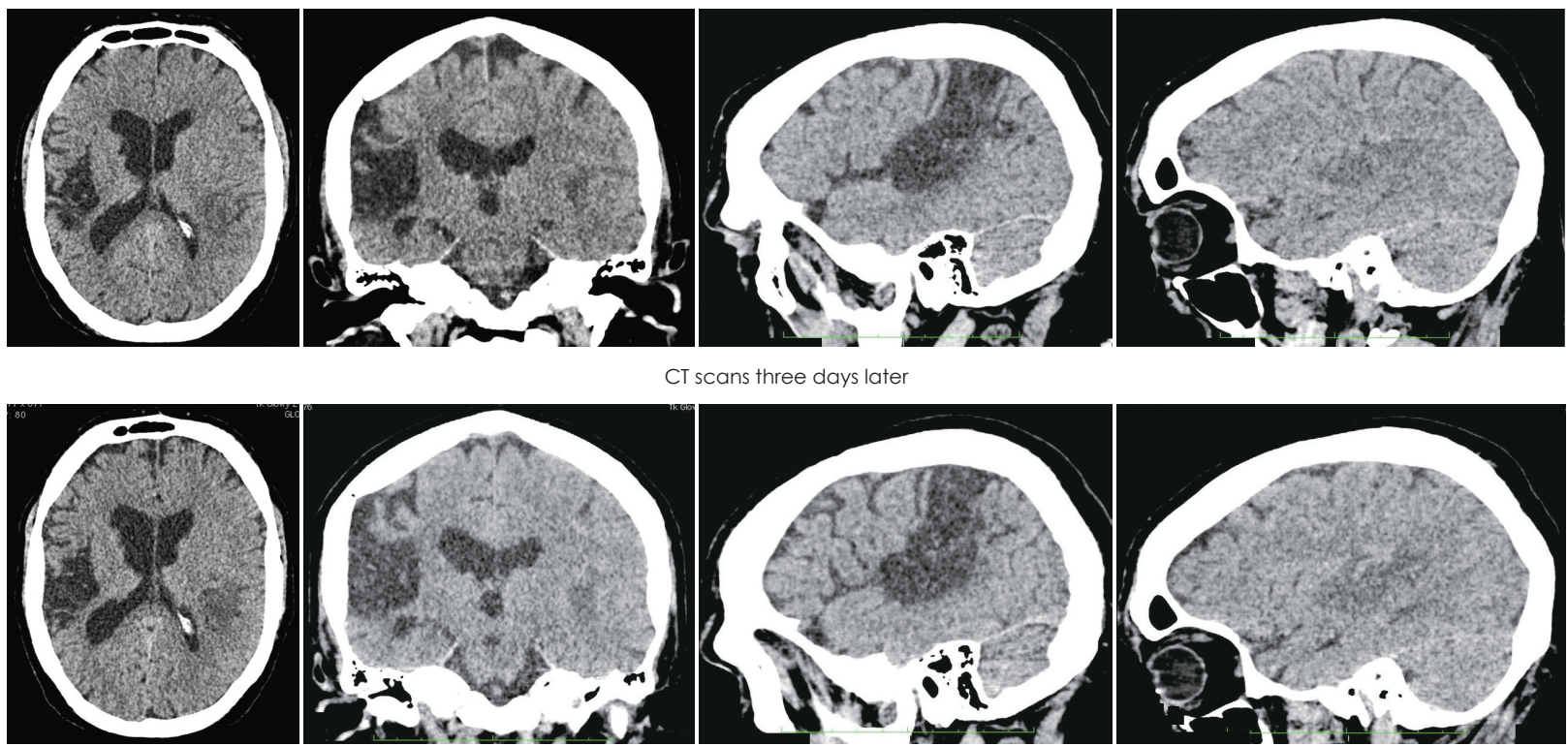

CT scans ten months later
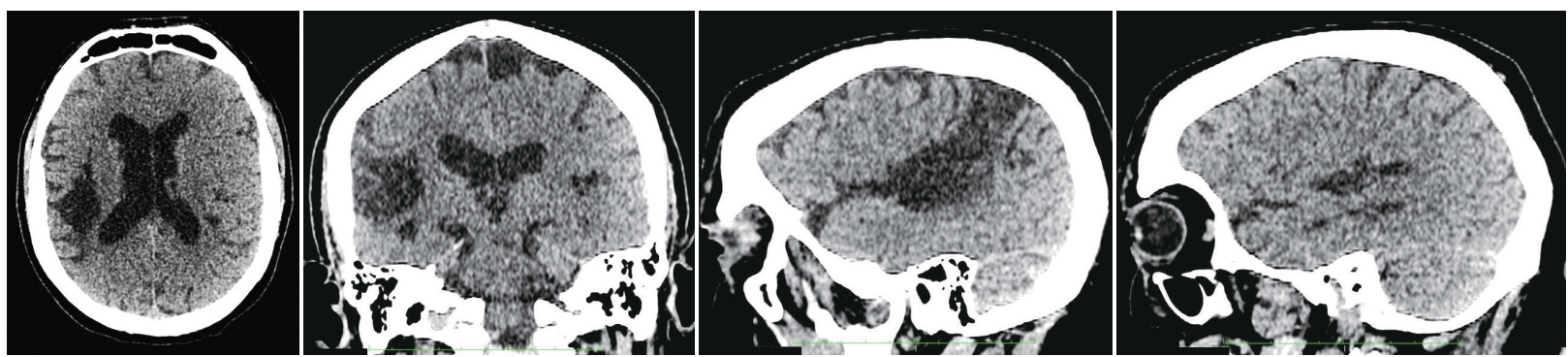

Fig. 3. CT of the brain performed on the day of the ischaemic stroke (first row), three days later (second row) and ten months later (third row). The scans show former massive ischaemic infarct lesion in the right hemisphere and evolution of the smaller acute ischemic infarct in the left temporal lobe. The first column presents axial section scans, second column coronal, third sagittal of the right hemisphere, and fourth sagittal of the left hemisphere.

The presently described acute ischaemic infarct in the left temporal lobe resulted in dramatic deafness experienced by the patient. Initially, the patient was unresponsive to all types of sounds, however, there were some auditory residual behaviors observed despite he completely denied hearing, which stays in line with the literature available [2,6]. In 10 months follow up, a small hearing improvement was observed in clinical features reported by the patient as well as the pure tone audiometry results. The patient claimed he could hear the environmental sounds and music, however, he still was unable to understand the spoken language. The pure tone audiometry showed pure tone thresholds improvement to the level of moderate hearing loss thresholds in the right ear and severe in the left. The DPOAEs, ABR results, and acoustic reflex findings showed normal results both at 3 and 10 months after the stroke, which is consistent with the normal functioning of the middle and inner ear and brain stem pathways. However, there was no single word discriminated in speech audiometry neither at 3 or 10 months after the stroke. Those symptoms suggest that cortical deafness in this patient evolved into word deafness so far (also known as verbal auditory agnosia) $[4,7,8]$. Word deafness is defined as an inability to understand spoken words despite intact hearing with better pure tone audiometry thresholds than would be expected by the degree of speech comprehension deficit [2,9]. As observed in the presented patient during the gradual, however still not complete, recovery of auditory function cortical deafness often evolves into word deafness. In the recovery process, the presented patient also was experiencing some degree of nonverbal deficits, including difficulties to identify the exact source of heard sounds or their exact nature. However, difficulty comprehending spoken words was the most distinctive deficit in his condition.

The improvement observed in the presented patient may be related to several factors. The neural connections in the area of the infarct might recover to some degree, and/or neu- 
ral fibers associated with hearing might redirect themselves around the damaged part of the temporal lobe, and/or some new neural fibers could be recruited and take over the functions of the damaged ones. The MLR and LLR potentials performed in the discussed patient might support this potential explanation. The Pa component of MLR was initially absent on both sides, however, appeared in MLR testing a few months later. In the beginning, the LLR's P1-N1 complex with wide N1 was observed on the left side and $\mathrm{P} 1$ with probable N1 on the right, and a few months later P1-N1 complex was visible on both sides, confirming objectively the improvement of patient's hearing, however, still they were somewhat compromised on both sides. The MLR and LLR results may support the improvement of hearing the sounds; however, they do not reflect any other signal processing like e.g. discrimination of sounds or comprehension of spoken words.

The event-related potentials were performed to evaluate some more complex signal processing abilities. The absent MMN confirmed above mentioned comprehension difficulties. In the literature, similar observations are described, however, the extend of abnormalities is dependent on the extent of the lesion in the cortical and subcortical parts of the brain $[3,12,13]$. As mentioned before, over 10 months after stroke the patient hearing improved to the level of environmental sounds and music hearing ability, however with some degree of problems with recognizing and processing the sounds. Despite that, his speech comprehension was still impaired. The new or recovered neural connections to the cortex may support the process for hearing improvement observed, however, further explanation may be as follows that they were not enough in number and did not achieve their neural synchrony to provide speech comprehension. The probable dyssynchronous input to the temporal lobes and associated structures would result in no event-related potentials disclosed in the patient. In other words, in this case, the cortex was probably deprived of most of the necessary signal input required to generate potentials such as MMN.

Recovery after stroke is still not fully understood phenomenon. There are few possible mechanisms i.e. modulation of close to lesion cortical structures and function, or modulation distant from the lesion with changes in ipsi- and/or contralateral hemisphere interaction, or shifting of cortical representational maps, or alterations in functional connectivity. Recovery could be spontaneous, which seems to be more probable in the presented case or induced by treatment for example physical therapy or stimulation therapies [14-16]. The understanding of the underlying recovery mechanisms comes from studies on patients or animals with mostly motor or verbal disturbances. There are only a few studies on patients suffering from ac- quired bilateral cortical deafness. Due to the patient's medical history and impossibility to perform more advanced imaging in this patient i.e. MRI and functional MRI, it is not possible to fully understand the nature of patients' partial recovery. It may be only speculated on which of the above mentioned recovery mechanisms played a more important role. Taking into account only the CT performed in the patient, one may speculate ischemic changes in the left Heschl's gyrus observed in CT scans lead to the modulation process of the nearby primary auditory cortex. It might be explained by noticed with time enlargement of the ischemic area with prolonged penumbra and probable increasing edema that could have lead to modulation of close to the lesion cortical structure and function. However, the improvement due to other distal brain modulation, for example, cross-modal synaptic plasticity from other brain regions cannot be completely excluded $[17,18]$. Regarding the old constant changes in the right hemisphere that were from the beginning larger than on the left side modulation changes or plasticity in this region was very unlikely to happen at the time of recovery from the second stroke.

In conclusion, cortical deafness presents unique diagnostic challenges as well as treatment difficulties. Patient recovery is unpredictable. In the presented case, cortical deafness was transient and evolved with time into more selective hearing disorder-word deafness, also known as verbal auditory agnosia. The extensive auditory electrophysiological testing performed and presented in this case report contributes to the understanding of the neural and functional changes in cortical deafness. It presents the evolution of changes in cortical deafness after ischaemic cerebrovascular event expressed as auditory evoked potentials starting from short through middle and long latency and ending with event-related potentials and supported by neuroimaging.

\section{Acknowledgments}

None

\section{Conflicts of interest}

The authors have no financial conflicts of interest.

\section{Author Contributions}

Conceptualization: Magdalena Lachowska. Data curation: Magdalena Lachowska. Formal analysis: Magdalena Lachowska, Agnieszka Pastuszka, Piotr Szczudlik. Investigation: Magdalena Lachowska, Agnieszka Pastuszka, Jacek Sokołowski, Piotr Szczudlik. Methodology: Magdalena Lachowska. Project administration: Magdalena Lachowska, Kazimierz Niemczyk. Supervision: Magdalena Lachowska, Kazimierz Niemczyk. Visualization: Magdalena Lachowska, Piotr Szczudlik. Writing — original draft: Magdalena Lachowska, Agnieszka Pastuszka, Piotr Szczudlik. Writing — review \& editing: Magdalena Lachowska, Jacek Sokołowski, Kazimierz Niemczyk. Approval of 
final manuscript: all authors.

\section{ORCID iDs}

Magdalena Lachowska https://orcid.org/0000-0003-3631-2551

Agnieszka Pastuszka https://orcid.org/0000-0002-8624-3358

Jacek Sokołowski https://orcid.org/0000-0001-9384-4189

Piotr Szczudlik https://orcid.org/0000-0003-4585-3851

Kazimierz Niemczyk https://orcid.org/0000-0003-2055-6615

\section{REFERENCES}

1) Silva J, Sousa M, Mestre S, Nzwalo I, Nzwalo H. Cortical deafness of following bilateral temporal lobe stroke. J Stroke Cerebrovasc Dis 2020;29:104827.

2) Musiek FE, Chermak GD, Cone B. Central deafness: a review of past and current perspectives. Int J Audiol 2019;58:605-17.

3) Ozdamar O, Kraus N, Curry F. Auditory brain stem and middle latency responses in a patient with cortical deafness. Electroencephalogr Clin Neurophysiol 1982;53:224-30.

4) Kaga K, Shindo M, Tanaka Y. Central auditory information processing in patients with bilateral auditory cortex lesions. Acta Otolaryngol Suppl 1997;532:77-82.

5) Musiek FE, Lee WW. Neuroanatomical correlates to central deafness. Scand Audiol Suppl 1998;49:18-25.

6) Garde MM, Cowey A. "Deaf hearing": unacknowledged detection of auditory stimuli in a patient with cerebral deafness. Cortex 2000;36: 71-80.

7) Brody RM, Nicholas BD, Wolf MJ, Marcinkevich PB, Artz GJ. Cortical deafness: a case report and review of the literature. Otol Neurotol 2013;34:1226-9.

8) Polster MR, Rose SB. Disorders of auditory processing: evidence for modularity in audition. Cortex 1998;34:47-65.

9) Pinard M, Chertkow H, Black S, Peretz I. A case study of pure word deafness: modularity in auditory processing? Neurocase 2002;8:40-55.

10) Koohi N, Vickers D, Chandrashekar H, Tsang B, Werring D, Bamiou DE. Auditory rehabilitation after stroke: treatment of auditory processing disorders in stroke patients with personal frequency-modulated (FM) systems. Disabil Rehabil 2017;39:586-93.

11) Engelien A, Huber W, Silbersweig D, Stern E, Frith CD, Döring W, et al. The neural correlates of 'deaf-hearing' in man: conscious sensory awareness enabled by attentional modulation. Brain 2000;123 Pt 3:532-45.

12) Musiek FE, Charette L, Morse D, Baran JA. Central deafness associated with a midbrain lesion. J Am Acad Audiol 2004;15:133-51; quiz 172-3.

13) Narayanan S, Majeed KA, Subramaniam G, Narayanan A, Navaf KM. A case of cortical deafness due to bilateral heschl gyrus infarct. Case Rep Med 2017;2017:6816748.

14) Cassidy JM, Cramer SC. Spontaneous and therapeutic-induced mechanisms of functional recovery after stroke. Transl Stroke Res 2017;8: 33-46.

15) Hordacre B, Moezzi B, Ridding MC. Neuroplasticity and network connectivity of the motor cortex following stroke: a transcranial direct current stimulation study. Hum Brain Mapp 2018;39:3326-39.

16) Kang N, Summers JJ, Cauraugh JH. Non-invasive brain stimulation improves paretic limb force production: a systematic review and meta-analysis. Brain Stimul 2016;9:662-70.

17) Glick H, Sharma A. Cross-modal plasticity in developmental and age-related hearing loss: Clinical implications. Hear Res 2017;343: 191-201.

18) Ding H, Qin W, Liang M, Ming D, Wan B, Li Q, et al. Cross-modal activation of auditory regions during visuo-spatial working memory in early deafness. Brain 2015;138:2750-65. 